\title{
The SiLuDrain Trial: a prospective randomized controlled trial comparing standard versus silver-impregnated lumbar drains
}

\author{
Martin Jakobs, MD, ${ }^{1}$ Sabrina Klein, MD, ${ }^{2}$ Tatjana Eigenbrod, MD, ${ }^{2}$ \\ Andreas W. Unterberg, MD, PhD, ${ }^{1}$ and Oliver W. Sakowitz, MD, PhD' \\ ${ }^{1}$ Department of Neurosurgery and ${ }^{2}$ Department of Infectious Diseases, Medical Microbiology, and Hygiene, University Hospital \\ Heidelberg, Heidelberg, Germany
}

\begin{abstract}
OBJECTIVE Catheter-associated cerebrospinal fluid (CSF) infection remains a serious event, especially for patients in neurocritical care units. The use of external ventricular drain (EVD) catheters impregnated with antimicrobial substances has led to a significant reduction of infection rates. This study was undertaken to compare the use of antimicrobial, silverimpregnated external lumbar drains (si-ELDs) and conventional ELDs.
\end{abstract}

METHODS Patients with an indication for ELD placement were randomized to receive either a conventional or an si-ELD catheter. Regular assessment for CSF infections and device-related complications was performed. Neurosurgeons placing the ELD rated the usability and handling of the catheter on a 6 -item ordinal performance scale (range: 1, very bad, to 5 , very good). All microorganisms isolated in this study were tested for silver-susceptibility via a catheter-roll method.

RESULTS A total of 48 patients were enrolled in the trial. The si-ELD catheters showed a nonsignificantly lower infection rate compared to conventional ones $(4.2 \%$ vs $16.7 \%, p=0.16)$. The majority of infections were caused by Staphylococcus species. Device-related complications occurred significantly less often with silver-impregnated-catheters than with conventional ones (8.3\% vs $37.5 \%, p=0.02)$. The usability was rated significantly better for si-ELDs $(p=0.003)$. Antimicrobial susceptibility was shown for si-ELDs against various Staphylococcus spp., but Candida parapsilosis and Escherichia coli were not affected by this antimicrobial agent.

CONCLUSIONS Silver-impregnated ELD catheters, which could potentially reduce the number of CSF infections, show significantly better properties in regard to handling and fewer device-related complications. Whether they are superior to antibiotic-impregnated catheters or a clinical regimen involving antibiotic prophylaxis remains to be proven.

Clinical trial registration no.: DRKS00013513 (Deutsches Register Klinischer Studien)

https://thejns.org/doi/abs/10.3171/2018.1.JNS181522

KEYWORDS CSF infection; external lumbar drain; silver-impregnated catheters; surgical technique; hydrocephalus; traumatic brain injury

$\mathrm{T}$ EMPORARY diversion of cerebrospinal fluid (CSF) is a common neurosurgical therapy for a variety of different diseases and states. Typically, CSF is either drained from the ventricles via an external ventricular drain (EVD), especially in cases of occlusive hydrocephalus, or drained from the thecal space via an external lumbar drain (ELD), particularly in cases of communicating hydrocephalus, CSF fistulas after trauma or surgery, or as a diagnostic test in patients with normal pressure hydrocephalus (NPH) $)^{3,6,17}$

Although the procedures of placing EVDs and ELDs are generally regarded as safe, catheter-associated CSF in- fections occurring after placement of EVDs/ELDs remain a significant clinical problem and may lead to worse patient recovery, longer hospital stays, and higher treatment costs.

Unlike blood-borne catheter-associated infections and urinary tract infections, there is no generally accepted definition for a catheter-associated CSF infection.

Accordingly, reported infection rates range widely from 0\%-32\% for $\mathrm{EVDs}^{9,16}$ and $1 \%-10 \%$ for ELDs. ${ }^{1,14}$

After antimicrobial central venous catheters were introduced and proven to reduce the rate of infection,,$^{15}$ this approach was adopted by the industry for EVDs and shunt catheters..$^{5,8,11,12}$

ABBREVIATIONS CFU = colony forming unit; CSF = cerebrospinal fluid; ELD = external lumbar drain; EVD = external ventricular drain; $\mathrm{NPH}=$ normal pressure hydrocephalus; $\mathrm{RCT}$ = randomized controlled trial; si-ELD = silver-impregnated ELD.

SUBMITTED November 26, 2017. ACCEPTED January 24, 2018.

INCLUDE WHEN CITING Published online June 15, 2018; DOI: 10.3171/2018.1.JNS181522. 
In general, antimicrobial catheters are impregnated either with antibiotics (single agent or a combination) or silver salts (e.g., silver sulfadiazine).

Although there have been several studies and randomized controlled trials (RCTs) that investigated the benefits of antimicrobial EVD catheters, ${ }^{11,22}$ there has not yet been a dedicated study comparing antimicrobial and conventional ELD catheters.

Furthermore, antimicrobial EVD catheters seem to be especially effective at preventing CSF infections with gram-positive bacteria. ${ }^{2}$ However, there are only sparse preclinical data available on the antimicrobial effects of si-catheters ${ }^{27}$ on different bacteria and fungi.

As this is a pilot study, the primary outcome is the comparison between conventional ELDs and silver-impregnated ELDs (si-ELDs) with regard to usability and handling, as tested with a questionnaire.

Secondary outcomes are the rate of infections for each ELD system and the rate of complications (both being defined by a set of criteria) and post hoc testing of all isolated microorganisms for silver susceptibility.

\section{Methods}

The SiLuDrain Trial (originally named the Silver-Coated Lumbar Drainage Trial) was conducted and planned as a monocentric open-label prospective randomized controlled trial to compare handling and infection and complication rates of conventional and si-ELD systems. This trial was registered with the German Registry of Clinical Trials (Deutsches Register Klinischer Studien; www.drks. de) as DRKS00013513.

\section{Patients}

Adults with an indication for ELD placement were eligible to participate in the study as long as they had not undergone ELD placement during the previous 3 months and did not have preexisting or suspected meningitis/CSF infection, known silver allergy, or known or suspected leptomeningeal spread of tumor cells. Concomitant placement of an EVD was not regarded as grounds for exclusion.

After it was determined that a patient met the criteria for inclusion, the patient or his/her legal representative was given oral and written information on the SiLuDrain study, was offered the opportunity for study participation, and was asked to provide written informed consent.

\section{Randomization}

Treatment allocation was randomized on a 1:1 ratio in randomization blocks of 4 as determined by a preexisting list created by a randomizer. Study participants were given a consecutive study number. Consecutively numbered, identical sealed envelopes containing the allocated ELD placement kit, the questionnaire, and a study timeline to be added to the patients' charts were prepared by a study nurse otherwise not involved with patient recruitment of this study.

After written informed consent was obtained, participating physicians retrieved the consecutively numbered sealed envelopes. Treatment allocation was therefore revealed to the physicians and patients only immediately before ELD placement.

\section{Sample Size}

Based on retrospective data, the expected infection rate with ELDs was estimated to be $7 \%$. Therefore, a trial adequately powered to show a reduction of $50 \%$ or more in CSF infections with si-ELDs would require more than 1000 patients to be included. In this pilot study, the enrollment period was limited to 1 year with an expected enrollment of 40 participants (20 in each study arm).

\section{Lumbar Drain Kits}

The standard lumbar drain kits used in the control group were Integra Ideaflow ELD (Integra) sets without any antimicrobial impregnation.

The silver-impregnated lumbar drain kits used in the study group were Spiegelberg Silverline ELD sets.

Both catheters had the same outer and inner diameters (1.6 $\mathrm{mm}$ and $0.8 \mathrm{~mm}$, respectively) as well as a length of $80 \mathrm{~cm}$.

A 14-gauge Tuohy needle was used to perform a lumbar puncture for catheter insertion in both groups.

Using their respective connectors, the ELDs were connected to the Neuromedex VentrEX CSF collection system.

\section{ELD Placement}

Participating resident neurosurgeons (year 1-6) had received an introduction to this study prior to enrollment of the first patient. Only residents who were already trained in ELD placement participated.

ELDs were placed in a sterile fashion on the patient's ward. Perioperative antibiotic prophylaxis was not performed. In conscious patients, local anesthetics were administered. Lumbar puncture was performed in the L4-5 or L3-4 interlaminar space and a 20- to 30-cm length of the ELD catheter was inserted through the Tuohy needle into the intrathecal space. The patency of all systems was checked before removing the Tuohy cannula and connecting the catheter to the CSF collection system. The catheter was secured by draping it to the back and flank of the patient. The CSF collection system was adjusted in height according to the volume of CSF to be drained per hour (standard: 5-10 ml/hour).

\section{ELD Assessment}

After ELD placement the performing resident neurosurgeon rated the individual steps and the overall placement on an ordinal scale, awarding 1 to 5 points $(1=$ very bad, 2 = bad, 3 = moderate, $4=$ good, 5 = very good), in a short questionnaire. Six items were rated: preparation of placement, lumbar puncture, inserting the catheter, fixation of the catheter, connection to the CSF collection system, and the overall rating of ELD placement.

\section{ELD Management}

Daily inspections of the puncture site were performed. CSF samples were obtained on the day of ELD placement and then every second day until removal. CSF samples were checked for white blood cell counts and concentrations of protein, glucose, and lactate. Furthermore, Gram staining and microbiological cultures were performed to check for infection. ELDs were removed upon the deci- 
TABLE 1. Definition of catheter-associated CSF infection

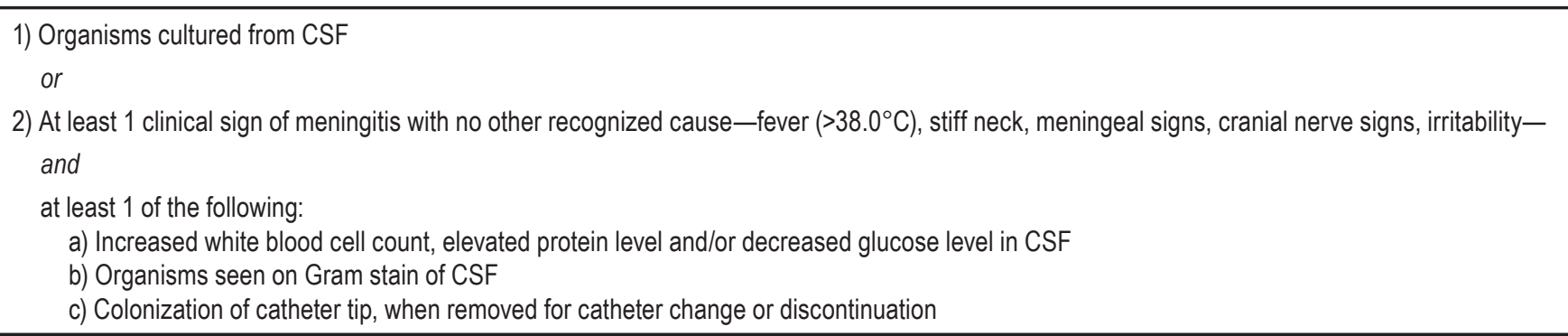

sion of the treating staff neurosurgeon and based on clinical criteria (see below). At the time of removal, the catheter tip was sent in for testing for microbial colonization.

\section{Definition of CSF Infection}

A slightly modified definition of the CDC definition ${ }^{10}$ for healthcare-associated meningitis/ventriculitis was used (Table 1).

\section{Indications for ELD Exchange}

A CSF infection defined as shown in Table 1 was judged as an indication for ELD exchange. Mechanical complications (dislocation, catheter tearing, catheter blockage) were also considered indications for ELD exchange.

Patients with an indication for an ELD exchange were not allowed to cross over into the other study group. In case the patient was regarded as not needing further temporary lumbar CSF drainage, the drain could be removed without replacement. Complications with the ELD systems were also documented (catheter occlusion, catheter dislocation, disconnection from the CSF collection system, catheter placement impossible).

\section{Antimicrobial Susceptibility Testing}

All isolated microbial strains from catheter tips and CSF samples were subjected to routine microbiological diagnostics as well as silver susceptibility testing to evaluate their susceptibility or resistance to the antimicrobial properties of ELDs.

The stored isolated strains were then analyzed for growth on silver-impregnated and conventional lumbar catheters as described previously. ${ }^{27}$ Conventional and silver-impregnated catheters of $4 \mathrm{~cm}$ length were separately placed in the bacterial suspension and incubated for 3 hours and then another 21 hours at $37^{\circ} \mathrm{C}$, and colonyforming units (CFUs) were counted at the end of both periods. ${ }^{7}$ A reduction of CFUs at the 24-hour time point compared to the 3-hour time point was regarded as a sign of antimicrobial susceptibility to silver in the absence of a similar reduction for the conventional catheter.

\section{Interpretation Criteria}

According to criteria defined by $\mathrm{Maki}^{18}$ for central venous catheters, an infection is suspected if 15 or more CFUs are grown after the catheter is rolled on an agar plate. Numbers of CFUs below 15 are regarded as evidence of contamination with skin flora.
As CSF is a sterile specimen, any detection of growth is regarded as relevant. However, due to manipulation of the system, contamination may occur when collecting the CSF. Therefore, results of microbiological cultures were interpreted with other signs of infection. Single cultivation in low amounts of bacteria from skin flora and without other signs of infection was regarded as indicating contamination.

\section{Statistics}

Statistics were calculated using SPSS version 23 (IBM Corp.). Descriptive statistics (statistical mean, median, range, and standard deviation) were used for demographic and clinical data (such as age, sex, indication for ELD, duration of ELD placement) from both study groups. To compare the study groups for statistical differences (such as in age, sex, or indication for ELD) the Student t-test was used. Differences in the numbers of CSF infections and the number of complications in the 2 groups were checked for statistical significant using the Pearson chi-square test. To compare the usability questionnaire scores for the 2 study groups and check for significant differences, the MannWhitney U-test was used. Statistical significance was defined as $\mathrm{p}<0.05$; confidence intervals were set at $95 \%$.

\section{Ethics}

This study was performed in accordance with the current version of the Declaration of Helsinki for ethical principles for medical research involving human subjects. The guidelines for good clinical practice were followed while planning and executing this study. The study was approved by the ethics committee of the medical faculty of the University of Heidelberg.

\section{Results}

\section{Patient Characteristics}

During the enrollment period a total of 54 patients were deemed eligible for the SiLuDrain trial. Six eligible patients were not included in the study: 2 of these patients reported having had an allergic reaction to silver and the other 4 refused to participate in a randomized trial and did not give informed consent after oral and written information about the nature of the study was given to them (also see Fig. 1).

A total of 48 patients were enrolled in this study, with 24 randomized to the si-ELD group (study group) and 24 randomized to receive a conventional ELD (control 


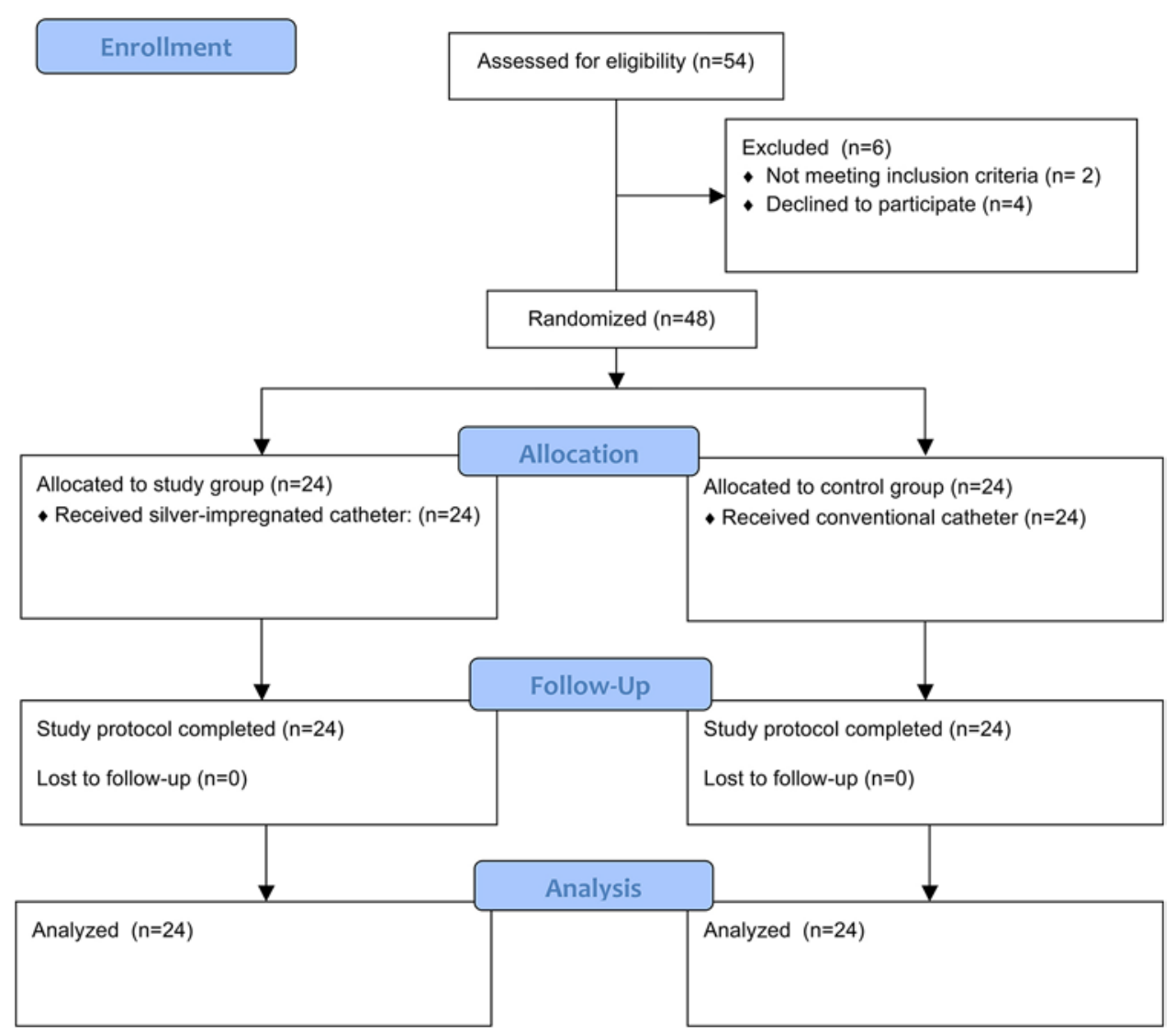

FIG. 1. SiLuDrain Trial flow diagram. Figure is available in color online only.

group). This result exceeded our expected sample size by $20 \%$. All patients could be included in the final analysis.

The study groups did not differ significantly with respect to demographic or clinical characteristics (Table 2). The indications for ELD placement were posthemorrhagic (communicating) hydrocephalus, CSF fistulas, and diag-

TABLE 2. Summary of demographic and clinical characteristics of the patient groups

\begin{tabular}{lcc}
\hline \multicolumn{1}{c}{ Characteristic } & si-ELD $(\mathrm{n}=24)$ & $\operatorname{Std} \operatorname{ELD}(\mathrm{n}=24)$ \\
\hline Sex, $\mathrm{n}(\%)$ & $9(37.5)$ & $10(41.7)$ \\
\hline Female & $15(62.5)$ & $14(58.3)$ \\
\hline Male & \\
\hline Age in yrs & 60.3 \\
\hline Mean & 60.6 & 14.2 \\
\hline SD & 14.7 & $28-84$ \\
\hline Range & $29-82$ & \\
\hline Indication for ELD, $\mathrm{n}(\%)$ & & $10(41.7)$ \\
\hline$\quad$ Posthemorrhagic hydrocephalus & $7(29.2)$ & $7(29.2)$ \\
\hline NPH testing & $8(33.3)$ & $7(29.2)$ \\
\hline CSF fistula & $9(37.5)$ & $7(29.2)$ \\
\hline EVD in place & $6(25.0)$ &
\end{tabular}

si-ELD = silver-impregnated ELD; Std ELD = standard (i.e., conventional) ELD. nostic tests. The variation of indications did not differ significantly between the 2 groups ( $p$ value range $0.37-0.76$, t-test).

\section{CSF Infections}

Catheter-associated CSF infections as defined previously were observed in $10.4 \%$ of cases. The number of catheter-associated CSF infections was lower in the study group $(1,4.2 \%)$ than in the control group $(4,16.7 \%)$, but the difference was not statistically significant $(\mathrm{p}=0.16$, chi-square test). Four of the 5 patients with CSF infections were female. In 4 cases the ELD was placed to treat posthemorrhagic hydrocephalus. One patient had received the ELD for treatment of a CSF fistula. None of the patients who underwent ELD placement for diagnostic tests developed a CSF infection.

CSF infections were diagnosed after a mean of 7.2 days (range 6-9 days) after ELD placement. The mean and median times that ELDs were placed for were exactly the same in both groups (mean 4.4 days, median 4.0 days, range 1-10 days).

In 3 of 5 patients, an EVD was in place at the time of ELD placement. In 3 patients, positive CSF cultures led to the diagnosis of CSF infection. Two more infections were determined by the combination of positive Gram stain and CSF pleocytosis or the combination of fever and colonization of the catheter tip. In all cases, different strains of staphylococci could be isolated either from CSF culture or 
TABLE 3. CSF infections and complications with ELD systems

\begin{tabular}{lll}
\hline \multicolumn{1}{c}{ Infection or Complication } & $\begin{array}{c}\text { si-ELD } \\
(\mathrm{n}=24)\end{array}$ & $\begin{array}{c}\text { Std ELD } \\
(\mathrm{n}=24)\end{array}$ \\
\hline CSF infection, $\mathrm{n}(\%)$ & $1(4.2)$ & $4(16.7)$ \\
\hline Complication, $\mathrm{n}(\%)$ & $2(8.3)^{*}$ & $9(37.5)^{*}$ \\
\hline Catheter occlusion & $1(4.2)$ & $3(12.5)$ \\
\hline Catheter dislocation & $1(4.2)$ & $2(8.3)$ \\
\hline Disconnection from CSF collection system & 0 & $2(8.3)$ \\
\hline Difficulty w/ catheter insertion $\dagger$ & 0 & $2(8.3)$ \\
\hline
\end{tabular}

* Statistically significant difference between groups $(p<0.05$, chi-square test).

$\dagger$ Requiring an additional lumbar puncture.

in relevant numbers on the tip of the catheter. In 3 cases, Staphylococcus epidermidis could be isolated. In another case, infection was caused by both S. warneri and S. cohnii. Staphylococcus hominis could be identified in another patient.

Only 2 ELD changes (both in the study group) occurred during this study. No ELD changes were performed in the control group. This difference was not statistically significant $(\mathrm{p}=0.15$, chi-square test). ELD changes were performed because of suspected bloodstream infections in critically ill patients. No CSF infections occurred. No proven bloodstream infection occurred in either group.

\section{ELD Complications}

The rate of device-related complications with ELD systems was $18.8 \%$ (Table 3). No severe complications (e.g., spinal epidural hematoma, nerve root injury, or intracranial hemorrhage) were detected. Only 2 complications occurred in the study group (8.3\%), whereas 9 complications were detected in the control group (37.5\%). This represents a significant difference in occurrence of devicerelated complications ( $\mathrm{p}=0.02$, chi-square test).

\section{ELD Usability}

In all cases, ELD assessment questionnaires were available (Table 4). The si-ELD systems were overall rated significantly better than the conventional ones $(p=0.003$, Mann-Whitney U-test). The ELD system from the study group had a higher median rating in each individual step of placing the ELD as well. Significant differences favoring si-ELD systems were detected for preparation of placement $(p=0.038)$, catheter insertion $(p=0.002)$, and catheter fixation $(\mathrm{p}=0.024)$, but not for lumbar puncture $(\mathrm{p}=0.116)$ or connection to the CSF collection system ( $p=0.667)$.

\section{Isolated Microorganisms and Antimicrobial Susceptibility Testing}

Microorganisms were isolated from 14 CSF samples or catheter tips; in 13 instances, the infecting organism was bacterial and in 1 instance it was fungal (Table 5). This includes the cases with proven CSF infection, cases with cultured microorganisms where contamination was assumed, and cases when catheters were removed as planned without the patient meeting criteria for catheter-
TABLE 4. Assessment of ELD usability

\begin{tabular}{lll}
\hline \multicolumn{1}{c}{ Parameter } & \multicolumn{1}{c}{$\begin{array}{c}\text { si-ELD } \\
(\mathrm{n}=24)\end{array}$} & $\begin{array}{c}\text { Std ELD } \\
(\mathrm{n}=24)\end{array}$ \\
\hline Preparation of placement & $4.0(\mathrm{good})^{*}$ & $3.0(\mathrm{avg})$ \\
\hline Lumbar puncture & $4.5(\mathrm{good} / \mathrm{v}$ good) & $4.0(\mathrm{good})$ \\
\hline Catheter insertion & $4.0(\mathrm{good})^{*}$ & $3.0(\mathrm{avg})$ \\
\hline Catheter fixation & $4.0(\mathrm{good})^{*}$ & $3.0(\mathrm{avg})$ \\
\hline Connection to CSF collection system & 4.0 (good) & $3.0(\mathrm{avg})$ \\
\hline Overall assessment & 4.0 (good) & $3.0(\mathrm{avg})$ \\
\hline
\end{tabular}

Avg = average $;$ = very.

Median scores are reported.

* Statistically significant difference between groups $(p<0.05$, Mann-Whitney U-test).

associated CSF infection. Only 1 case of infection with gram-negative rods (Escherichia coli) occurred. All other isolated organisms were gram-positive cocci, with S. epidermidis being the most common organism.

The 3-hour results show confluent and dense growth for all microorganisms in the isolate solutions containing the conventional catheters and those containing the silverimpregnated catheters, proving successful cultivation.

After 24 hours some samples showed reduced growth with both the conventional and the silver-impregnated catheter, but comparison of the range of reduction showed that the silver-impregnated catheter was superior to the conventional one in 10 of 14 cases (71.4\%). In 2 cases (one fungal and one gram-negative sample), neither of the solutions (i.e., the one containing the si-ELD or the one containing the conventional catheter) showed any reduction in growth. In 2 cases, the solution containing the silverimpregnated catheter showed less growth reduction compared to the conventional one. Interestingly these cases were 1 of the 4 cases of S.epidermidis and 1 of the 3 cases of S. haemolyticus.

\section{Discussion}

This study is - to our knowledge-the first RCT to investigate and compare conventional versus si-ELD catheters. Furthermore, this is also the first trial to document device-related complication rates for and elucidate the antimicrobial properties of silver-impregnated catheters.

As far as our rate of infection is concerned, $10.4 \%$ is toward the upper end of the range reported in the literature. ${ }^{1,14}$ This rate is higher than our own infection rate prior to this study (7\%). This difference is most likely explained by the fact that, like others, we did not have defined and strict criteria on how to determine that a patient had an ELD-associated CSF infection in our regular clinical practice.

Definitions for a catheter-associated CSF infection differ among authors. ${ }^{9,10}$ In most publications dealing with catheter-associated CSF infections, only a positive culture of a microorganism from a CSF sample is regarded as definitive evidence of an infection.

Only very recently the Infectious Diseases Society of America (IDSA) published practice guidelines for "healthcare-associated ventriculitis and meningitis." 25 
TABLE 5. Antimicrobial properties of si-ELD catheters with regard to bacterial and fungal organisms isolated and cultured from CSF or catheter tips

\begin{tabular}{|c|c|c|c|c|c|c|}
\hline Specimen No. & Organism & Std ELD, 3 Hrs & Std ELD, $24 \mathrm{Hrs}$ & si-ELD, 3 Hrs & si-ELD, $24 \mathrm{Hrs}$ & Growth Reduction w/ Silver \\
\hline 1 & C. parapsilosis & $>300$ & $>300$ & $>300$ & $>300$ & No \\
\hline 2 & S. epidermidis & $>300$ & 80 & $>300$ & 0 & Yes \\
\hline 3 & S. haemolyticus & $>300$ & $>300$ & $>300$ & 0 & Yes \\
\hline 4 & S. epidermidis & $>300$ & $>300$ & $>300$ & 0 & Yes \\
\hline 5 & S. hominis & $>300$ & 50 & $>300$ & 0 & Yes \\
\hline 6 & S. lentus & $>300$ & 7 & $>300$ & 2 & Yes \\
\hline 7 & S. epidermidis & $>300$ & $>300$ & $>300$ & 0 & Yes \\
\hline 8 & S. cohnii & $>300$ & $>300$ & $>300$ & 0 & Yes \\
\hline 9 & S. warneri & $>300$ & $>300$ & $>300$ & 0 & Yes \\
\hline 10 & S. epidermidis & $>300$ & 16 & $>300$ & 80 & No \\
\hline 11 & S. hominis & $>300$ & 30 & $>300$ & 6 & Yes \\
\hline 12 & S. haemolyticus & $>300$ & 2 & $>300$ & 30 & No \\
\hline 13 & S. haemolyticus & $>300$ & 40 & $>300$ & 0 & Yes \\
\hline 14 & E. coli & $>300$ & $>300$ & $>300$ & $>300$ & No \\
\hline 15 & S. aureus ATCC 25923 (control) & $>300$ & $>300$ & $>300$ & 26 & Yes \\
\hline
\end{tabular}

Data are numbers of CFUs; $>300$ CFUs indicates confluent growth.

These guidelines include all scenarios in which catheters are placed in the CSF space (EVDs, ELDs, ventriculoperitoneal shunts, and intrathecal pumps). Although there is no singular definition of a healthcare-associated CSF infection, the authors give a very nuanced guideline and recommendations of different strengths (weak, moderate, strong) with regard to clinical symptoms, findings in serum and CSF, and microbiological findings. All of the criteria that we utilized to define a CSF infection in this study are mentioned in this guideline, with CSF cultures being declared as the most important test to determine a CSF infection.

In our study, positive CSF cultures occurred in only $60 \%$ of our cases rated as infections. In 2 more cases, the combination of clinical signs of meningitis and CSF changes or a positive Gram stain led to the clinical definition of a catheter-associated CSF infection.

As the number of overall publications regarding ELDs is much smaller than for EVDs, a dedicated rate of devicerelated complications was not available in the literature. Our detected rate of $18.8 \%$, however, shows that patients with ELDs require special observation whether they are bedridden in an intensive care unit or mobile in a regular ward.

It should be noted that all infections in this study occurred in patients who either had intracranial surgery or had posthemorrhagic hydrocephalus. In only 1 of the 5 cases was ELD placement the first opening of the dura.

None of the patients with diagnostic indications for ELD placement (in most cases, suspected NPH) developed an infection. Not only did these patients not have another opening of the dural space before, but ELDs were usually placed for no longer than 72 hours, as this currently represents the method with the highest diagnostic value for $\mathrm{NPH} \cdot{ }^{17}$ None of the infections in our study occurred prior to day 6. This is in accord with the findings of Liang et al., ${ }^{14}$ who describe ELD placement for longer than 4 days to be a risk factor for infection in patients with after subarachnoid hemorrhage.

We saw a significantly lower rate of complications with silver-impregnated catheters. Although both ELDs used in this study had the same length as well as the same inner and outer diameter and both were placed using a 14-gauge Tuohy needle, there are differences to be noted. The conventional catheter must be placed using a guidewire, whereas the silver-impregnated one is more rigid and can be placed without any additional aids. This fact may be reflected in the significantly better usability score given for the silver-impregnated catheter in the "catheter insertion" category, as well as the occurrence of 2 complications involving catheter insertion with the need for an additional lumbar puncture in the control group.

Another aspect leading to 2 complications was the connection to the CSF collection system. Silver-impregnated catheters were rated nonsignificantly better than conventional ones for this step. Tight and durable connections to the CSF collection system can prevent disconnection and the resulting contact of CSF with the patient's environment, which would pose a risk for infection. While the conventional ELD has a connector that is sutured on, the silver-impregnated one has a Luer lock-like system, which did not lead to any disconnection in our study.

It should be noted that in our study CSF samples were obtained every other day while the catheter was in place to get a better overview of changes in CSF pleocytosis and growth of microorganisms. As every manipulation of a CSF drainage system can be a potential risk for infection, this is not recommended anymore. ${ }^{9}$ It is advised to only obtain CSF samples when there is strong clinical suspicion of CSF infection, which also has become common practice in our department. This and a catalog of other means have been shown to reduce the rate of infections associated with external CSF drains. ${ }^{13}$

One controversial consideration is prophylactic anti- 
biotic treatment with external CSF drains. In our study, the choice was up to the neurosurgeon in charge. Two of our 5 infections occurred in patients who were at that time receiving antibiotic treatment for respiratory tract infections. Prophylactic antibiotic treatment when placing a CSF drain is no longer recommended..$^{913}$ Whether it is advisable to give a single dose of an antibiotic prior to ELD placement as performed by Leverstein et al. and as recommended prior to EVD placement is currently unclear. ${ }^{13}$

Regarding the results of meta-analyses on antimicrobial EVD catheter use, it seems reasonable to assume that antimicrobial catheters are superior to conventional ones in terms of infection rates. However, it is so far not clear whether silver impregnation conveys a stronger advantage than antibiotic impregnation. ${ }^{2,5,26}$ To date, there are more studies published for catheters with antibiotic-impregnation and therefore the data may be interpreted as stronger. Nevertheless, a recent German survey among all major neurosurgical centers showed that si-EVDs are used more often compared to ones with antibiotic impregnation. ${ }^{3}$ Moreover, no dedicated ELD catheter with antibiotic impregnation is available on the market.

Testing silver-impregnated drains for growth reduction after inoculation with the isolated microorganisms showed that growth of gram-positive bacteria was especially inhibited. Out of 12 tested Staphylococcus isolates, the growth of 10 isolates was inhibited by the silver-impregnated drainage systems. Whether there is less activity against gram-negative organisms or fungi is not clear, as only one $E$. coli isolate and one Candida parapsilosis isolate was tested. Previous work by Zschaler reported growth reduction of 2 gram-negative reference strains, one E. coli and one Pseudomonas aeruginosa, as well as of 2 yeasts: $C$. glabrata and $C$. albicans. Therefore, it may be a strainspecific effect. However, the majority of catheter-related infections are caused by gram-positive bacteria, especially staphylococci, ${ }^{2}$ and no single antibiotic-impregnated drain cannot prevent infection by all potential pathogens.

Our findings on the range of silver susceptibility and silver resistance of bacteria confirm but also exceed the known effects published earlier. ${ }^{27}$ However, our results do not elucidate what kind of reduction of growth can clinically prevent a CSF infection, as multiple factors come into play.

The mechanism of action has not been fully determined. Silver ions bind to various structures of the cell and may thereby disturb cellular function and integrity. ${ }^{4,21}$ The antimicrobial activity of silver does not target a specific cellular structure, and therefore it is able to exert its effect on gram-positive and gram-negative bacteria as well as fungi. Nevertheless, resistance to silver has also been observed. ${ }^{21,23}$ It is interesting though, that in 2 cases single strains of S.epidermidis and S. haemolyticus did not show this effect. It is up for speculation whether genetic alterations or functional changes lead to resistance.

A correlation between the use of antibiotic-impregnated catheters and infection with methicillin-resistant $S$. aureus (MRSA) has been described. ${ }^{12}$ Silver-impregnated drains may offer the benefit of a broad antimicrobial activity against gram-negative and gram-positive bacteria as well as fungi.

Silver compounds and conventional antibiotics may exert a synergistic effect. ${ }^{19-21}$ Especially for the treatment of intraventricular infections, as some antibiotics, such as vancomycin and gentamycin, may be administered directly into the ventricle. ${ }^{24}$ It is not known whether silver nanoparticles or compounds are shed from the catheter and may be dissolved in the CSF.

This is the first study to provide combined information on patient characteristics, isolated microorganisms, and correlation with in vitro antimicrobial effect of the silverimpregnated drains on microbial isolates obtained from patients' CSF and/or catheter tips. Interestingly, one $S$. epidermidis isolate from one patient with infection in the study group was not inhibited by the silver-impregnated catheter in vitro. The other isolates from patients in the control group were inhibited by the silver-impregnated drains. One can speculate as to whether the use of si-ELDs would have prevented infection in these particular patients.

Our study does suffer from several limitations. First and foremost is the sample size. An adequately powered study would have required inclusion of more than 1000 patients, which would only be possible in the setting of a large multicenter trial, presumably with industry sponsorship. We can therefore not claim a significant reduction in catheter-associated infection with si-ELDs. Furthermore, patients with ELD placement for diagnostic tests may be neglected in a follow-up study, as it seems that catheter placement for less than 72 hours is not associated with a relevant risk of infection.

Although randomized and controlled, our study was conducted as an open trial. This is due to the fact that no manufacturer was able to supply us with both conventional and silver-impregnated catheters and a neutral packaging. In particular, the resident neurosurgeons who placed the ELDs were aware of the type of catheter they were using in each case. This might have induced a certain degree of bias with regard to the assessment of ELD usability. Still, our study provided some valuable information, as the first study to compare conventional and si-ELD catheters.

\section{Conclusions}

In our study, we showed a reduction in the number of catheter-associated CSF infections in patients who received si-ELDs compared with a control group treated with conventional ELDs, but we were unable to demonstrate statistical significance, presumably due to the small sample size. Usability of the si-ELDs was rated significantly better than that of the conventional ELDs, and significantly fewer complications occurred in the si-ELD group. Staphylococci were the most common microorganisms associated with CSF infections. Silver-impregnated catheters show in most cases a higher in vitro reduction of growth of grampositive bacteria. Patients with ELDs should be given close attention to further minimize the risk of infection or other device-related complications.

\section{Acknowledgments}

We would like to thank Dr. Tom Bruckner from the Institute for Medical Biometrics and Informatics (IMBI), Heidelberg, for advice and support regarding statistical design and randomization of this study. 


\section{References}

1. Al-Tamimi YZ, Bhargava D, Feltbower RG, Hall G, Goddard AJ, Quinn AC, et al: Lumbar drainage of cerebrospinal fluid after aneurysmal subarachnoid hemorrhage: a prospective, randomized, controlled trial (LUMAS). Stroke 43:677-682, 2012

2. Atkinson RA, Fikrey L, Vail A, Patel HC: Silver-impregnated external-ventricular-drain-related cerebrospinal fluid infections: a meta-analysis. J Hosp Infect 92:263-272, 2016

3. Cinibulak Z, Aschoff A, Apedjinou A, Kaminsky J, Trost HA, Krauss JK: Current practice of external ventricular drainage: a survey among neurosurgical departments in Germany. Acta Neurochir (Wien) 158:847-853, 2016

4. Clement JL, Jarrett PS: Antibacterial silver. Met Based Drugs 1:467-482, 1994

5. Cui Z, Wang B, Zhong Z, Sun Y, Sun Q, Yang G, et al: Impact of antibiotic- and silver-impregnated external ventricular drains on the risk of infections: a systematic review and meta-analysis. Am J Infect Control 43:e23-e32, 2015

6. D’Anza B, Tien D, Stokken JK, Recinos PF, Woodard TR, Sindwani R: Role of lumbar drains in contemporary endonasal skull base surgery: meta-analysis and systematic review. Am J Rhinol Allergy 30:430-435, 2016

7. European Committee on Antimicrobial Susceptibility Testing: Breakpoint tables for interpretation of MICs and zone diameters. Version 3.1. EUCAST.org. (http://www.eucast. org/fileadmin/src/media/PDFs/EUCAST_files/Breakpoint_ tables/Breakpoint_table_v_3.1.pdf) [Accessed February 21, 2018]

8. Fichtner J, Güresir E, Seifert V, Raabe A: Efficacy of silverbearing external ventricular drainage catheters: a retrospective analysis. J Neurosurg 112:840-846, 2010

9. Fried HI, Nathan BR, Rowe AS, Zabramski JM, Andaluz N, Bhimraj A, et al: The insertion and management of external ventricular drains: an evidence-based consensus statement. Neurocrit Care 24:61-81, 2016

10. Horan TC, Andrus M, Dudeck MA: CDC/NHSN surveillance definition of health care-associated infection and criteria for specific types of infections in the acute care setting. Am J Infect Control 36:309-332, 2008 (Erratum in Am J Infect Control 36:655, 2008)

11. Keong NC, Bulters DO, Richards HK, Farrington M, Sparrow OC, Pickard JD, et al: The SILVER (Silver Impregnated Line Versus EVD Randomized trial): a double-blind, prospective, randomized, controlled trial of an intervention to reduce the rate of external ventricular drain infection. Neurosurgery 71:394-404, 2012

12. Konstantelias AA, Vardakas KZ, Polyzos KA, Tansarli GS, Falagas ME: Antimicrobial-impregnated and -coated shunt catheters for prevention of infections in patients with hydrocephalus: a systematic review and meta-analysis. J Neurosurg 122:1096-1112, 2015

13. Leverstein-van Hall MA, Hopmans TEM, van der Sprenkel JW, Blok HE, van der Mark WA, Hanlo PW, et al: A bundle approach to reduce the incidence of external ventricular and lumbar drain-related infections. J Neurosurg 112:345-353, 2010

14. Liang H, Zhang L, Gao A, Li Y, Jiang Z, Hu F, et al: Risk factors for infections related to lumbar drainage in spontaneous subarachnoid hemorrhage. Neurocrit Care 25:243-249, 2016

15. Lorente L: Antimicrobial-impregnated catheters for the prevention of catheter-related bloodstream infections. World J Crit Care Med 5:137-142, 2016

16. Lozier AP, Sciacca RR, Romagnoli MF, Connolly ES Jr: Ventriculostomy-related infections: a critical review of the literature. Neurosurgery 62 (Suppl 2):688-700, 2008
17. Mahr CV, Dengl M, Nestler U, Reiss-Zimmermann M, Eichner G, Preuß M, et al: Idiopathic normal pressure hydrocephalus: diagnostic and predictive value of clinical testing, lumbar drainage, and CSF dynamics. J Neurosurg 125:591-597, 2016

18. Maki DG, Weise CE, Sarafin HW: A semiquantitative culture method for identifying intravenous-catheter-related infection. N Engl J Med 296:1305-1309, 1977

19. Panáček A, Smékalová M, Kilianová M, Prucek R, Bogdanová K, Večeřová R, et al: Strong and nonspecific synergistic antibacterial efficiency of antibiotics combined with silver nanoparticles at very low concentrations showing no cytotoxic effect. Molecules 21:E26, 2015

20. Panáček A, Smékalová M, Večeřová R, Bogdanová K, Röderová M, Koláŕ M, et al: Silver nanoparticles strongly enhance and restore bactericidal activity of inactive antibiotics against multiresistant Enterobacteriaceae. Colloids Surf B Biointerfaces 142:392-399, 2016

21. Percival SL, Bowler PG, Russell D: Bacterial resistance to silver in wound care. J Hosp Infect 60:1-7, 2005

22. Pople I, Poon W, Assaker R, Mathieu D, Iantosca M, Wang E, et al: Comparison of infection rate with the use of antibioticimpregnated vs standard extraventricular drainage devices: a prospective, randomized controlled trial. Neurosurgery 71:6-13, 2012

23. Silver S: Bacterial silver resistance: molecular biology and uses and misuses of silver compounds. FEMS Microbiol Rev 27:341-353, 2003

24. Tunkel AR, Hartman BJ, Kaplan SL, Kaufman BA, Roos KL, Scheld WM, et al: Practice guidelines for the management of bacterial meningitis. Clin Infect Dis 39:1267-1284, 2004

25. Tunkel AR, Hasbun R, Bhimraj A, Byers K, Kaplan SL, Michael Scheld W, et al: 2017 Infectious Diseases Society of America's clinical practice guidelines for healthcare-associated ventriculitis and meningitis. Clin Infect Dis 64:34-65, 2017

26. Wang X, Dong Y, Qi XQ, Li YM, Huang CG, Hou LJ: Clinical review: Efficacy of antimicrobial-impregnated catheters in external ventricular drainage-a systematic review and meta-analysis. Crit Care 17:234, 2013

27. Zschaler R: Testing of the Antimicrobial Effect of Catheter Tubing with a Roll Culture Method. (http://www. spiegelberg.de/documents/Zschaler.pdf) [Accessed February $21,2018]$

\section{Disclosures}

The authors report no conflict of interest concerning the materials or methods used in this study or the findings specified in this paper.

\section{Author Contributions}

Conception and design: Jakobs, Sakowitz. Acquisition of data: Jakobs, Klein, Eigenbrod. Analysis and interpretation of data: Jakobs, Klein, Eigenbrod, Sakowitz. Drafting the article: Jakobs, Klein, Sakowitz. Critically revising the article: Jakobs, Klein, Unterberg, Sakowitz. Reviewed submitted version of manuscript: Jakobs, Klein, Unterberg, Sakowitz. Approved the final version of the manuscript on behalf of all authors: Jakobs. Administrative/ technical/material support: Unterberg, Sakowitz. Study supervision: Unterberg, Sakowitz.

\section{Correspondence}

Martin Jakobs: University Hospital Heidelberg, Germany. martin. jakobs@med.uni-heidelberg.de. 